\title{
Stable Set and Voting Rules
}

\author{
Klaus Kultti* \\ Paavo Miettinen ${ }^{\dagger}$ \\ Department of Economics \\ University of Helsinki \\ FPPE \& Department of Economics \\ University of Helsinki \\ Department of Economics, University of Helsinki \\ Discussion Papers, No. 582:2003 \\ ISSN 1459-3696 \\ ISBN 952-10-1232-3
}

November 20, 2003

\begin{abstract}
We consider a voting situation where a society has to decide on the rule to use when choosing among two alternatives in the uncertain future. Our analysis is related to the set up of Barbera and Jackson (2001). While they consider finite societies in our set up the economy has an infinite amount of agents. We define a binary dominance relation over the set of decision rules and determine the von Neumann and Morgenstern stable set of voting rules. It turns out that the stable set always exists and is unique in the infinite economy's case. The stable set is however not a singleton. Additionally the stable set coincides with the set of self-stable voting rules that is the solution concept used by Barbera and Jackson (2001).
\end{abstract}

${ }^{*}$ Department of Economics, University of Helsinki, P.O. Box 17, Arkadiankatu 7, FIN-00014 University of Helsinki, Finland. E-mail: kkultti@valt.helsinki.fi

${ }^{\dagger}$ Finnish Post Graduate Program in Economics and Department of Economics, University of Helsinki, P.O. Box 17, Arkadiankatu 7, FIN-00014, University of Helsinki, Finland. E-mail: paavo.a.miettinen@helsinki.fi 


\section{Introduction}

Barbera and Jackson (BJ henceforth) (2001) raise the question on how a society decides which rules it uses to decide on issues. What makes this problem intriguing is that, as a rule, decisions are based on some rules, and this immediately raises the question on how these rules are chosen and an infinite regress ensues. A satisfactory answer to the problem seems to require some kind of fixed point property of the rule: A good rule should select itself.

Without any other structure the problem seems hard to get in touch with, and BJ consider a specific situation where the society consist of a finite number of agents who must make a choice between two alternatives $a$ and $b$. The former is a status quo alternative, and the latter is a challenger that may replace the status quo. The potential rules are just numbers $s \in\{1, \ldots, n\}$ where $n$ is the number of agents in the society. A rule $s$ means that in a voting $b$ against $a$ the former replaces the latter if it gets at least $s$ votes. The dichotomous choice environment is convenient as it precludes strategic voting.

The agents in the society have preferences that are captured by a number $p_{i} \in[0,1]$ which is the probability that the agent favours the change of status quo ( $b$ over $a$ ). One can then determine the induced preferences over the set of decision rules which are used in to choose between $a$ and $b$. The agents have to come to an agreement on the rule before they know whether they favour the status quo or the change.

Now it is possible to put any rule in the position of a status quo and consider which rule would be chosen if the status quo rule were used in deciding this. BJ define the notion of self-stability which captures the idea that a rule can maintain itself if it is the status quo and it is put against all the other rules in a dichotomous choice situation. There may be a multitude of self-stable rules or such can also fail to exist.

BJ consider finite societies, and the calculations determining the preferences over rules and selfstability are involved. In this article we rid ourselves of the problems of finiteness by considering a society with finitely many types where each type is of some measure. We show that the nonexistence of self-stable rules ceases but there is always a multiplicity of them.

We think that instead of defining a single-valued solution concept that has some kind of inherent stability property it is useful to take one step backwards and define a dominance relation between 
the rules. Using this we determine the von Neumann-Morgenstern stable set which we propose as a solution concept. It turns out that there is always a unique stable set. In a sense we do not get rid of the multiplicity problem as the stable set is not a singleton but it shows in an interesting way how the rules relate to each other. The dominance relation we use says roughly the following: A rule $s$ dominates another rule $s^{\prime}$ if using rule $s^{\prime}$ rule $s$ would be chosen.

Moving from a finite society to a continuum society we lose some interesting features that BJ have in their article. For instance, in the finite society it is the relative values, not the absolute values of $p_{i} \in[0,1]$, that determine the agents preferences over rules. With very many agents the number of agents that support change is almost certain, and the agents' preferences become such that all the rules above the expected number of supporters are equally good, and all the rules below this number are equally good. On the upside we gain in the sense that we get much more clear cut results than in the finite society.

In section 2 we present the basics of the model, and in section 3 we determine the stable set and the self-stable voting rules. In section 4 we compare the stable rules given by the notions of the stable set and self-stability. In section 5 we present the conclusions.

\section{The Model}

In the society there exists a state of affairs $a$ which is called the status quo. Then there also exists an alternative state of affairs $b$ which may be chosen to be the new status quo. The decision between $a$ and $b$ is be made by a vote in some future date. There exists a continuum of voters in the society, and each voter's preference is represented by a number $p_{i} \in[0,1]$. This tells the probability that she will vote for the alternative $b$ when the election date comes up. There is a finite number of types of voters numbered so that $p_{1}<p_{2}<\ldots<p_{n}$. The measure of type $i$ voters is $\alpha_{i}$ and the measure of all agents is unity, $\sum_{i=1}^{n} \alpha_{i}=1$. With this specification there are exactly

$$
B=\sum_{i=1}^{n} \alpha_{i} p_{i}
$$

agents who vote for $b$ when it is staged against the status quo $a$. Note that in the infinite society the relative probabilities do not matter any more; the only important thing is whether a voter will 
vote for $b$ with a probability that is less or more than one half. This means that we are roughly ${ }^{1}$ back in the simple case that BJ call a dichotomous society, and where they show that self-stable voting rules always exist. ${ }^{2}$

Definition 1 A voting rule $s$ is a number $s \in(0,1]$.

If at least measure $s$ of voters vote for the alternative $b$ then $b$ is selected. Otherwise the status quo $a$ remains. Utility for voter $i$ from status quo $a$ is given by

$$
\begin{aligned}
& U_{i}(a \mid i \text { favors } a)=1 \\
& U_{i}(a \mid i \text { favors } b)=0 .
\end{aligned}
$$

The utility for voter $i$ from $b$ is given by

$$
\begin{aligned}
& U_{i}(b \mid i \text { favors } b)=1 \\
& U_{i}(b \mid i \text { favors } a)=0 .
\end{aligned}
$$

Next we determine the voters' utilities when some voting rule $s$ is used. The status quo $a$ is maintained if $s>B$. The alternative $b$ is chosen as a result of the vote when a rule $s \leq B$ is used. Consider a voting rule that requires at least a measure $s$ of supporters. The utility for a voter $i$ from this voting rule is defined by

$$
\begin{aligned}
& u_{i}(s)=p_{i} U_{i}(a \mid i \text { favors } b)+\left(1-p_{i}\right) U_{i}(a \mid i \text { favors } a), \text { when } s>B \\
& u_{i}(s)=p_{i} U_{i}(b \mid i \text { favors } b)+\left(1-p_{i}\right) U_{i}(b \mid i \text { favors } a), \text { when } s \leq B
\end{aligned}
$$

Then we have that if $k$ is the largest probability such that $p_{k} \leq \frac{1}{2}$ the voters with $i \leq k$ want a rule $s>B$ and voters $i=k+1$ with probabilities $p_{k+1}>\frac{1}{2}$ want a rule $s \leq B$.

\footnotetext{
${ }^{1}$ See conclusion for further details.

${ }^{2}$ It is also unnecessary to have a finite number of types.
} 


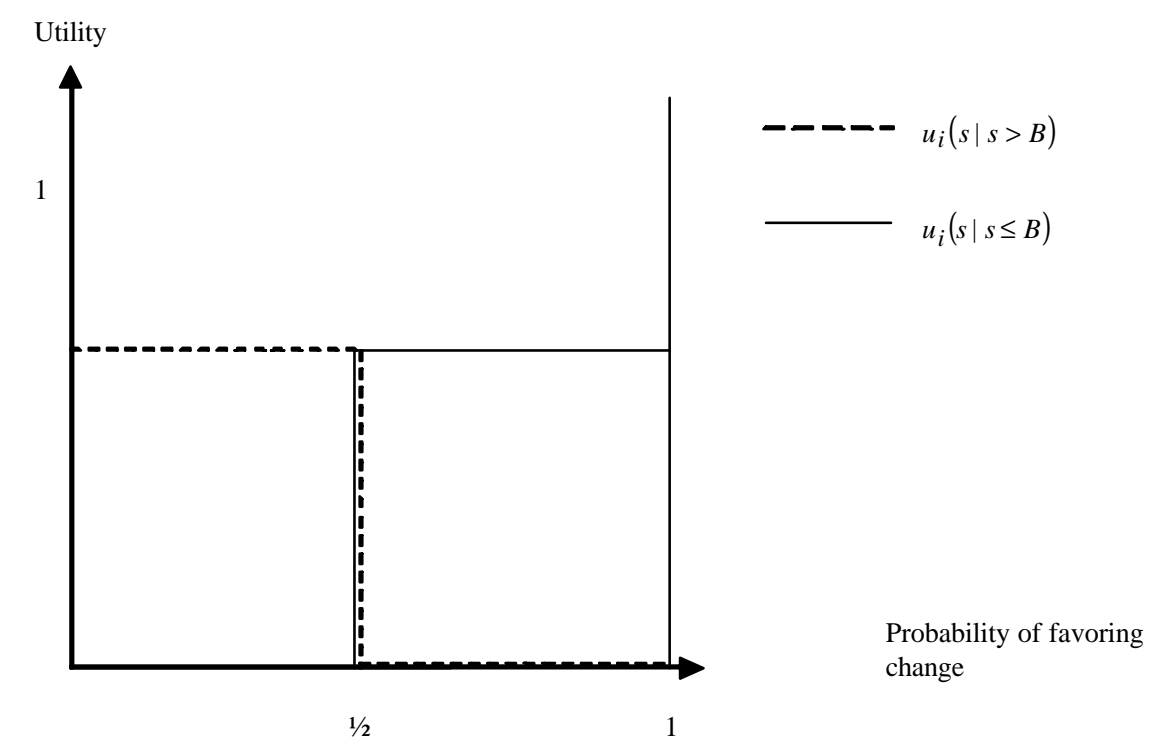

Utility functions

\section{$3 \quad$ Stable set and self-stability}

We consider two different notions of stable voting rules. First we introduce a dominance relation between different voting rules and consider whether there exists a von Neumann-Morgenstern stable set. We find that the stable set exists and it is unique. Second in the continuum case self-stable voting rules exist, like in the case of a dichotomous society in BJ, but there is not hope of uniqueness.

Definition 2 We denote the measure of all types $i$ that satisfy some property $\pi$ as

$$
\mu\{i \mid \pi\}=\sum_{i \in\{1, \ldots, n\}: \text { property } \pi \text { is true }} \alpha_{i} .
$$

Let's now define a dominance relation.

Definition 3 A voting rule $s$ dominates voting rule $s^{\prime}$ if $\mu\left\{i \mid U_{i}(s)>U_{i}\left(s^{\prime}\right)\right\} \geq s^{\prime}$. We denote this dominance relation as $s \Delta s^{\prime}$.

Thus if the decision makers have to decide between the rules $s$ and $s^{\prime}$ then the rule $s^{\prime}$ is dominated by the rule $s$ if it receives more than a measure $s^{\prime}$ of votes. That is, when $s^{\prime}$ is the rule 
used to make decisions, then there exists at least one rule $s$ such that $s$ gets selected over $s^{\prime}$. This, of course, would suggest that such a rule is very poor since it can't sustain itself. Next we give the definition of a von Neumann-Morgenstern stable set:

Definition $4 A$ set $S$ is internally stable if $\forall s, s^{\prime} \in S, \neg\left(s^{\prime} \Delta s\right)$ and $\neg\left(s \Delta s^{\prime}\right)$ holds.

Definition $5 A$ set $S$ is externally stable if $\forall s \notin S \exists s^{\prime} \in S: s^{\prime} \Delta s$.

Definition 6 A set $S S$ is the stable set if it is both internally and externally stable.

Thus the internal stability requires that the rules in the stable set do not dominate each other and the external stability says that all rules that do not belong to the stable set are dominated by some rule in the stable set. Additionally we give the definition of self-stable voting rule:

Definition 7 (Barbera and Jackson (2001))

$A$ voting rule $s$ is self-stable if for all $s^{\prime} \neq s$

$$
\mu\left\{i \mid U_{i}\left(s^{\prime}\right)>U_{i}(s)\right\}<s .
$$

From above it is clear that here rule $s$ has a status quo position. That is, rule $s$ is used when deciding about rules. A self-stable voting rule $s$ must sustain itself when any rule $s^{\prime}$ is brought up against the self-stable voting rule.

If for all types $i \leq k$ the probability of preferring the change is $p_{i} \leq \frac{1}{2}$ and for all types $i>k+1$ the probability is $p_{i}>\frac{1}{2}$, then the measure of types that prefer rules that are larger than $B$ is $\underline{s}=\sum_{i=1}^{k} \alpha_{i}$. Hence all "small" rules with $s \leq B$ get $1-\underline{s}$ votes against rules $s^{\prime}>B$ and all "large" rules $s>B$ get $\underline{s}$ votes against rules $s^{\prime} \leq B$. We can now calculate the measures of proponents for different rules:

A rule $s \leq B$ dominates $s^{\prime}>B$ if

$$
\mu\left\{i \mid U_{i}(s)>U_{i}\left(s^{\prime}\right)\right\} \geq s^{\prime} \Leftrightarrow 1-s \geq s^{\prime} .
$$

Similarly a rule $s>B$ dominates $s^{\prime} \leq B$ if 


$$
\mu\left\{i \mid U_{i}(s)>U_{i}\left(s^{\prime}\right)\right\} \geq s^{\prime} \Leftrightarrow \underline{s} \geq s^{\prime}
$$

Additionally if $s, s^{\prime} \leq B$, or $s, s^{\prime}>B$ then

$$
\mu\left\{i \mid U_{i}(s)>U_{i}\left(s^{\prime}\right)\right\}=0 .
$$

We now show that with the dominance relation $\Delta$ a stable set exists. The first proposition proves the two cases where all voters prefer either the status quo or the alternative. In the second proposition the proof is organized in six parts. First we find a set of rules that are not dominated by any rules. It should be noted that none of these rules may be left outside of the stable set since otherwise the external stability would be violated. Then we show that no matter which rule we pick outside this set we always find a rule from this set that dominates the rule outside. Then, as a consequence of this construction, the stable set is also unique. Note also that we do not have to check whether rules that are "on the same side" of $B$ dominate each other since by (11) they are equivalent from the voters' point of view.

Proposition 1 With the dominance relation $\Delta$ a stable set exists if either $p_{i}>\frac{1}{2}$ or $p_{i} \leq \frac{1}{2}$ for all $i \in\{1, \ldots, n\}$.

Proof. Let $p_{i} \leq \frac{1}{2}$ for all $i \in\{1, \ldots, n\}, B$ is defined as above and $\underline{s}=\sum_{i=1}^{n} \alpha_{i}=1$. Then (9) cannot hold for any $s^{\prime}>B$. Now any set $(0, \tilde{s}] \subset(0, B]$ can not be a stable set since there does not exist a rule $s<B$ such that $s \Delta s^{\prime}$ would hold for any $s^{\prime}>B$. In this case all voters prefer large rules and the stable set is $(B, 1]$.

Similarly it can be shown that the stable set exists and is unique when $p_{i}>\frac{1}{2}$ for all $i \in\{1, \ldots, n\}$. In this case the stable set is $(0, B]$.

Proposition 2 If for all $i \in\{1, \ldots, k\}, p_{i} \leq \frac{1}{2}$ and for $i \in\{k+1, \ldots, n\}, p_{i}>\frac{1}{2}$, then a stable set exists.

Proof. i.) Suppose that $1-\underline{s} \leq \underline{s}<B$ then the stable set is $S S=(\underline{s}, 1]$

We first note that all the rules $s^{\prime}$ in the set $[0, \underline{s}]$ are dominated by the rules $s$ in $(B, 1]$ since by (10) it holds that $s \geq s^{\prime}$. Now let $s \in(s, B]$ and $s^{\prime} \in(B, 1]$ then by (9) $s \Delta s^{\prime}$ would imply that 
$1-\underline{s} \geq s^{\prime}>B$ which is a contradiction. Similarly $s^{\prime} \Delta s$ would imply by (10) that $\underline{s} \geq s^{\prime}>B$ which is also a contradiction. Then all the rules that are not dominated are in the set $(\underline{s}, 1]$. If the set that contains all the rules that are not dominated contains a rule $s$ that belongs to the stable set then this implies that the whole set belongs to the stable set. Let's pick a rule $s$ from the set $(B, 1]$. Since these rules dominate all rules in the set $[0, \underline{s}]$ then $s$ and also $(s, 1]$ belong to the stable set. As the rules in $[0, \underline{s}]$ are dominated we have that the stable set is $(\underline{s}, 1]$.

ii.) Suppose now that $\underline{s} \leq 1-\underline{s}<B$ then the stable set is $S S=(\underline{s}, 1]$. The proof is analogous to the case $i$.

iii.) Suppose that $s<B \leq 1-s$ then the stable set is $S S=(s, B] \cup(1-s, 1]$.

Let $s^{\prime} \in(0, s]$ and $s \in(1-s, 1]$. Then $s^{\prime} \Delta s$ would imply by (9) that $1-s \geq s$ which is a contradiction. Similarly $s \Delta s^{\prime}$ implies by (10) that $\underline{s} \geq s^{\prime}$ which holds.

Let now $s^{\prime} \in(B, 1-\underline{s}]$ and $s \in(\underline{s}, B]$. Then $s^{\prime} \Delta s$ would imply by (10) that $\underline{s} \geq s$ which is a contradiction. Similarly $s \Delta s^{\prime}$ implies by (9) that $1-\underline{s} \geq s^{\prime}$ which holds.

Additionally we note that when $s^{\prime} \in(0, \underline{s}]$ and $s \in(B, 1-\underline{s}]$ we have that $s^{\prime} \Delta s$ holds since (9) gives us $1-\underline{s} \geq s$ and also $s \Delta s^{\prime}$ holds since by (10) $\underline{s} \geq s^{\prime}$. Hence the rules in $(0, \underline{s}] \cup(B, 1-\underline{s}]$ are dominated.

Now let $s \in(\underline{s}, B]$ and $s^{\prime} \in(1-\underline{s}, 1]$. Then $s^{\prime} \Delta s$ would require by (10) that $\underline{s} \geq s$ which clearly does not hold. Similarly $s \Delta s^{\prime}$ requires by (9) that $1-\underline{s} \geq s^{\prime}$ which does not hold either. Thus all the rules that are not dominated are in the set $(s, B] \cup(1-s, 1]$. Since we showed above that the rules in $(s, B]$ dominate the rules in $(B, 1-s]$ and that the rules in $(1-s, 1]$ dominate the rules in $(0, \underline{s}]$ we have established that the stable set is $(\underline{s}, B] \cup(1-\underline{s}, 1]$.

iv.) Suppose that $1-\underline{s} \leq B \leq \underline{s}$ then the stable set is $(B, 1]$.

Let's pick any rule $s \in(B, 1]$ then by (10) we have that $s \Delta s^{\prime}$ since $\underline{s} \geq s$ is true for all $s^{\prime} \in[0, B]$. Similarly (9) gives us that $s^{\prime} \Delta s$ can not hold since $1-\underline{s}<s$ is true for all $s \in(B, 1]$. Then the rules that are not dominated are in the set $(B, 1]$. These rules dominate the rules in $[0, B]$ and thus the stable set is $(B, 1]$.

v.) Suppose that $B<1-\underline{s} \leq \underline{s}$ then the stable set is $(1-\underline{s}, 1]$.

Let $s \in(1-\underline{s}, 1]$ and $s^{\prime} \in[0, B]$ then by (10) we have that $s \Delta s^{\prime}$ since $\underline{s} \geq s^{\prime}$ is true for all $s^{\prime} \in[0, B]$. Similarly $s^{\prime} \Delta s$ would imply by (9) that $1-s \geq s \in(1-s, 1]$ which is a contradiction. 
Note that for rules $s \in(B, 1-\underline{s}]$ and rules $s^{\prime} \in[0, B]$ both $s^{\prime} \Delta s$ and $s \Delta s^{\prime}$ hold since by (9) $1-\underline{s} \geq s$ and by $(10) s \geq s^{\prime}$. Then the rules that are not dominated are in the set $(1-\underline{s}, 1]$. These rules dominate the rules in $[0,1-\underline{s}]$ and thus the stable set is $(1-\underline{s}, 1]$.

vi.) Suppose that $B \leq \underline{s}<1-\underline{s}$. Then the stable set is $(1-\underline{s}, 1]$. The proof is analogous to the case v.

Combining propositions 1 and 2 the stable set always exists and is unique.

Next we show that the self-stable voting rules always exists and that the set of self-stable rules coincides with the rules in the stable set.

Proposition 3 A self-stable voting rules always exists and the set of self-stable rules coincides with the stable set.

Proof. A rule $s$ is self-stable if for all $s^{\prime} \neq s$ inequality (8) holds. Then rule $s$ is not self-stable if there exists a rule $s^{\prime}$ such that $\mu\left\{i \mid U_{i}\left(s^{\prime}\right)>U_{i}(s)\right\} \geq s \Leftrightarrow \exists s^{\prime} \neq s: s^{\prime} \Delta s$. Then the requirement of self-stability is equivalent to the requirement that no rules dominate $s$. Thus to find the set of self-stable rules one needs to look for the rules that are not dominated. Since in Propositions 1 and 2 we showed that all the rules in the stable-set are not dominated it follows immediately that the set of self-stable rules coincide with the stable set.

\section{Comparison of stable set and the set of self-stable voting rules and interpretations}

There are no differences between the rules in the stable set and the set of self-stable voting rules. In all cases above the set of self-stable rules and the stable set coincide. Thus we conclude that the dominance relation with the von Neumann-Morgestern stable set is equivalent to the notion of self-stable voting rules in this set up with a continuum of agents.

There are two forces that affect the stable set. First, the voting rules have different inherent properties with respect to our dominance relation $\Delta$. "Very small" rules are easy to get rid of since when they are in a status quo position only a small number of votes are required to replace them. In contrast, "very large" rules in status quo position require a large number of votes to be 
replaced. It is because of these properties that in all cases above the stable set contains no "very small" rules and on the other hand it always contains the "very large" rules. Secondly, the number of proponents of large and small rules, of course, affects which rules are easily replaced and which are not.

In the case where $1-\underline{s} \leq \underline{s}<B$ we have that the proponents of the large rules are a larger group than the proponents of the small rules. However the proponents of the large rules are too few to exclude the small rules $(\underline{s}, B]$ from the stable set that is $(\underline{s}, 1]$. The proponents of the small rules are, however, too small a group to rule out any large rules. That's why the stable set contains all large rules and the set $(s, B]$ of small rules. The fact that the stable set contains both small and large rules reflects the weakness of the voters that prefer large rules. If there were more than $B$ voters that prefer the large voting rules then the stable set would not contain any small rules. This is essentially the case iv. The interpretations are similar in the case where the roles of $1-\underline{s}$ and $\underline{s}$ are reversed.

In the case where $\underline{s}<B \leq 1-\underline{s}$ the proponents of the small rules are a larger group than the proponents of the large rules. Now the proponents of the small rules fail to exclude the large rules $(1-\underline{s}, 1]$ from the stable set. In order to exclude all large rules from the stable set all voters would have to be in favor of the small rules as is shown in proposition 1. Similarly the proponents of the large rules are too small a group to exclude the small rules $(\underline{s}, B]$ from the stable set. Since the inherent properties of the voting rules make it easy to rule out the "very small" rules and difficult to exclude the "very large" rules the stable set is $(s, B] \cup(1-s, 1]$. Note that the proponents of change are a larger group ex ante than they will be ex post $B \leq 1-s$. This also gives rationale for the fact that also large rules belong to the stable set. Some proponents of the small rules will eventually vote for the status quo.

Finally in the case where both $1-\underline{s}$ and $\underline{s}$ are larger than $B$ the stable set contains only large rules that is $S S=(1-\underline{s}, 1]$. This is because the proponents of the large rules are numerous enough to exclude all small rules from the stable set. Since the proponents of the small rules are also quite large the proponents of the large rules cannot include rules $B<s \leq 1-\underline{s}$ to the stable set. Even if the proponents of the small rules were a larger group than the proponents of the large rules 
(case vi. above), only large rules, that preserve the status quo, belong to the stable set. This is because some voters that prefer small rules are bound to find themselves voting for the status quo. The reason is same as above: the proponents of change are a larger group ex ante than they will eventually be ex post.

\section{Conclusion}

A move from the finite agent environment of BJ to an environment with a continuum of agents and a finite number of types allows us to understand some aspects of the notion of self-stability better. In particular, when the number of agents increases the importance of the relative probabilities decreases, and the agents' preferences depend just on whether their ex-ante probability of favouring the change is more or less than one half.

Another point of view to the problem of which rules one can expect to be chosen is got by considering a dominance relation between the rules, and determining the von Neumann-Morgenstern stable set. This turns out to exist in all cases, and it is also unique. In addition using the dominance relation that we gave in the beginning one gets exactly the same set of decision rules as one would get when using the notion of self-stability. The set up of a continuum society is not exactly equivalent to dichotomous society since the set of self-stable rules and stable set coincide in one setting but fail to do so in another ${ }^{3}$. However the way to characterize the set of self-stable rules with our dominance relation works also in the finite case. In our opinion the dominance relation is more fundamental than the notion of self-stability and we see this close relationship as justifying the self-stability notion given that one perceives our dominance relation as a reasonable one. The dominance relation could well be defined in some other way. Another natural candidate for the dominance relation that we considered is not as good as $\Delta$, as there may be a multiplicity of stable sets. This relation is defined as follows: A voting rule $s$ dominates voting rule $s^{\prime}$ if $\mu\left\{i \mid U_{i}(s)>U_{i}\left(s^{\prime}\right)\right\} \geq s$. The interpretation is that a rule $s$ dominates another rule $s^{\prime}$ if $s$ happens to be the status quo, and $s$ selects itself. Note that this does not eliminate the possibility that $s$ would also select rule $s^{\prime}$.

\footnotetext{
${ }^{3}$ The exact calculations concerning the characterization of the stable set and the set of self-stable rules are available from the authors.
} 
Consider a situation where for all $i \in\{1, \ldots, k\}, p_{i} \leq \frac{1}{2}$ and for $i \in\{k+1, \ldots, n\}, p_{i} \geq \frac{1}{2}$. Now $(0, B]$ is a stable set if $\sum_{i=k+1}^{n} \alpha_{i} \geq B$. Set $(B, 1]$ is a stable set if $\sum_{i=1}^{k} \alpha_{i}>B$. As $B=\sum_{i=1}^{n} p_{i} \alpha_{i}$ these two conditions can be satisfied simultaneously. An example is a society with only two types where $p_{1}=0.3$ and $p_{2}=0.6, \alpha_{2}=1-\alpha_{1}$ and $\frac{4}{7} \geq \alpha_{1}>\frac{6}{13}$.

Finally we note that all the analysis here is based on the voting situation where one alternative, be it a real one or a rule, is a status quo and it is staged against a challenger. This is quite specific, and in a way bypasses a lot that is interesting when one tries to figure out what kind of rules emerge in a situation where no rule is given any special status. The problem is that most other formulations seem to lead to a game situation where it is not easy determine the induced preferences over the rules, while in the setting of this article, the agents always vote truthfully.

\section{References}

[1] Badger, W. W. (1972): "Political Individualism, Positional Preferences and Optimal DecisionRules" in Probability Models of Collective Decision Making, edited by R.G. Niemi and H. F. Weisberg, Merrill Publishing: Columbus Ohio.

[2] Barbera, S. and Jackson, M. O.: "Choosing How to Choose: Self-Stable Majority Rules and Constitutions", working paper at http://www.hss.caltech.edu/〜jacksonm/Jackson.html. 